\title{
M2 macrophage infiltration into tumor islets leads to poor prognosis in non-small-cell lung cancer
}

This article was published in the following Dove Press journal:

Cancer Management and Research

\author{
Lili Cao ${ }^{1,2, *}$ \\ Xiaofang Che ${ }^{1,2, *}$ \\ Xueshan Qiu ${ }^{3}$ \\ Zhi Li ${ }^{1,2}$ \\ Bowen Yang ${ }^{1,2}$ \\ Shuo Wang ${ }^{1,2}$ \\ Kezuo Hou ${ }^{1,2}$ \\ Yibo Fan ${ }^{1,2}$ \\ Xiujuan $\mathrm{Qu}^{1,2}$ \\ Yunpeng Liu ${ }^{1,2}$ \\ 'Department of Medical Oncology, The \\ First Hospital of China Medical University, \\ Shenyang I I000 I, People's Republic of \\ China; ${ }^{2}$ Key Laboratory of Anticancer \\ Drugs and Biotherapy of Liaoning Province, \\ The First Hospital of China Medical \\ University, Shenyang I I000 I, People's \\ Republic of China; ${ }^{3}$ Department of \\ Pathology, College of Basic Medical \\ Sciences of China Medical University, \\ Shenyang I I000I, People's Republic of \\ China \\ *These authors contributed equally to this \\ work
}

Background: Lung cancer is the leading cause of cancer-related death worldwide. Although the macrophages can affect the development of tumor, the contribution of macrophages to the prognosis of non-small-cell lung cancer (NSCLC) is still controversial. Moreover, anti-PD-1 therapy can redirect macrophages from an M2 to an M1 phenotype, suggesting that tumor PD-L1 may affect the prognostic role of macrophages. Therefore, in this study, we aimed to display a macrophage landscape to clarify the function of macrophages, considering the localization and polarization of the macrophages, and evaluate the effect of M2 macrophages and tumor PD-L1 in combination on the prognosis of NSCLC.

Methods: We performed multiplex quantitative immunofluorescence staining of pan-cytokeratin (CK), CD68, CD163, PD-L1, and DAPI on one tissue specimen simultaneously from 137 NSCLC patients.

Results: M2 macrophages, involved marginM2 (M2 macrophages in tumor stroma), and centralM2 (M2 macrophages infiltrating into tumor islets) increased as the tumor stage increased. More macrophages were found in lung squamous cell carcinoma (LUSC) patients, patients with wild-type EGFR, and smokers than in patients with lung adenocarcinoma (LUAD), patients with EGFR mutations, and non-smokers. Infiltration of centralM2 was an independent prognostic factor of poor overall survival (OS) and disease-free survival (DFS) for NSCLC patients $(P<0.05)$, which was superior to total macrophages and total M2 macrophages. Moreover, patients with centralM2 ${ }^{\text {less }} \mathrm{PD}-\mathrm{L1}^{-}$tumors showed the best OS and DFS, while the patients with centralM2 ${ }^{\text {more }} \mathrm{PD}-\mathrm{L}^{+}{ }^{+}$tumors showed the worst OS and DFS, and the two groups with centralM $2{ }^{\text {less }} \mathrm{PD}-\mathrm{L}_{1}{ }^{+}$and centralM $2{ }^{\text {more }} \mathrm{PD}-\mathrm{L}^{-}{ }^{-}$were in the middle $(P=0.002,0.034$, respectively) Conclusion: Tumor islet-infiltrating M2 macrophages influence the prognosis of NSCLC patients. The analysis of M2 macrophages and tumor PD-L1 in combination may enhance the accuracy of prognostic prediction. This study provides a new understanding of macrophages in the development of NSCLC through the analysis of macrophage landscape.

Keywords: macrophage, microenvironment, polarization, non-small-cell lung cancer, prognosis, PD-L1

\section{Introduction}

Lung cancer is the most common type of cancer and remains the leading cause of cancer-related death worldwide. ${ }^{1,2}$ Smoking, gene mutation, and reprogramming of the immune microenvironment are critical factors that may affect malignancy in lung cancer. The vital role of the tumor immune microenvironment (TIME) has attracted increasing attention as progress with immunotherapy in lung cancer is made. ${ }^{3-5}$

Among the various immune cells present in the TIME, macrophages are the predominant population of tumor-infiltrating immune cells. ${ }^{6}$ Macrophages may differentiate into either pro-inflammatory (M1) or anti-inflammatory

(M2)
Correspondence: Yunpeng Liu; Xiujuan Qu First Hospital of China Medical University, District, Shenyang I I000I, People's

Republic of China

Tel +862483282312

Fax +86248328 2543

Email ypliu@cmu.edu.cn;

xiujuanqu@yahoo.com 
macrophages, and each type plays different roles in the development of cancer. M1 macrophages mainly function in killing pathogens and tumor cells, whereas M2 macrophages function in promoting tumor development. ${ }^{7-15}$ Although the role of macrophages in tumor progression is well known, the effect of macrophages on tumor prognosis is still contradictory. Previous studies have reported that macrophage infiltration is associated with poor prognosis in lung cancer, ${ }^{16,17}$ breast cancer, ${ }^{18}$ and prostate cancer, ${ }^{19}$ whereas it is associated with good prognosis in prostate $^{20}$ and colon cancer. ${ }^{21}$ In addition, some other studies reported that no relationship was found between macrophages and prognosis of lung cancer. ${ }^{22,23}$ Those contradictory results may be due to the opposing roles of M1 and M2 macrophages, ${ }^{24-27}$ as well as the differing roles of macrophages in distinct tumor locations. ${ }^{28-30}$ In addition, the traditional immunohistochemistry regularly used in previous studies could not simultaneously exhibit the polarization states and distribution of macrophages objectively and accurately, which may also be an important reason for the contradictory results. Therefore, in this study, we aimed to clarify the function of macrophages with regard to both their localization and polarization in patients with NSCLC using multiplex quantitative immunofluorescence staining.

It is known that programmed death receptor 1 (PD-1), an inhibitory receptor mainly expressed on $\mathrm{T}, \mathrm{B}$, and $\mathrm{NK}$ cells that inhibits antitumor immunity, is also expressed on tumor-associated macrophages (TAMs). ${ }^{31}$ Recently, it was reported that anti-PD-1 therapy could redirect macrophages from an M2 to an M1 phenotype in osteosarcoma, ${ }^{32}$ suggesting that PD-1/PD-L1 signaling may affect macrophage reprogramming in the TIME. Moreover, although PD-L1 plays an important immunosuppressive role in tumor development, the prognostic effect of tumor PD-L1 has not been consistent in previous studies. ${ }^{33-41}$ One of the possible reasons was speculated that tumor PD-L1 may affect the prognosis by altering macrophage function. Therefore, PDL1 and macrophages together may affect the prognosis of cancer. However, no previous studies have examined the effect of macrophages and tumor PD-L1 in combination on tumor prognosis.

In this study, we investigated the effects of macrophage number, localization, and polarization on the development of non-small-cell lung cancer (NSCLC) using multiple quantitative fluorescence staining. Additionally, we also examined the combined effects of tumor PD-L1 expression and macrophage infiltration on the prognosis of NSCLC.
These results provide a new understanding of the importance of macrophages in the development of NSCLC.

\section{Materials and methods Patients}

One hundred and thirty-seven patients diagnosed with NSCLC between 2012 and 2014 were enrolled as the following criteria: NSCLC with stage I-III; no neoadjuvant therapy before surgery; received standard adjuvant therapy after operation. All the above patients were followed up at the First Hospital of China Medical University. The median follow-up period was 55.6 months (range, 8-75 months), 65 patients relapsed $(47.44 \%)$, and 35 patients $(25.55 \%)$ died during this period, with a 5-year overall survival (OS) probability of $34.31 \%$. The study was approved by the Ethics Committee of China Medical University (No. 2017-236), and all procedures were conducted in accordance with ethical principles. Clinical information of all patients was retrieved from the Hospital Information System.

\section{Multiple quantitative fluorescence staining}

Multiple quantitative fluorescence staining was performed with the Opal 7-Color Manual IHC Kit (NEL811001KT, PerkinElmer Inc., Waltham, MA, USA) according to the protocol of the manufacturer, which allows for microwave treatment to remove primary and secondary antibodies while retaining an intact fluorescent signal. This process is repeated until all antigens have been stained with their respective fluorophores.

The antibodies were diluted as follows:

Anti-PD-L1 (\#13,684, clone E1L3N, CST), 1:300, Anti-CD68 (ZM-0060, zhongshanjinqiao, China), 1:200, Anti-CD163 (ZM-0428, zhongshanjinqiao, China), 1:400, Pan-cytokeratin (CK) (MAB-0671, Maixin Biotech, China), working fluid,

DAPI (FP1490A, PerkinElmer), working fluid.

\section{Image analysis}

Multiple quantitative fluorescence staining data were collected by Mantra Quantitative Pathology Workstation (PerkinElmer, CLS140089) and analyzed by PerkinElmer inForm Analysis software. Each scanned image was visually examined by a pathologist, and regions/samples with staining artifacts and with large necrotic areas were excluded. Image processing comprised the steps of training session and image analysis session. The training 
session included manual annotation of three distinct types of region (tumor, stroma, and blank areas) performed by a pathologist. A machine-learning algorithm built into the inForm software was used to create the tissue segmentation algorithm. Cell segmentation was performed on the basis of the nuclear DAPI staining.

We defined tumor PD-L1 positive as PD-L1-positive tumor cells accounting for more than $1 \%$ of the total cells and defined the macrophage as high infiltration if the macrophage density is greater than the median and the low infiltration is opposite.

\section{Statistical analyses}

Cell density among subgroups was analyzed using nonparametric test for continuous variables. Survival curves were determined using the Kaplan-Meier method and compared using the log-rank test. COX regression analysis with continuous variables was performed to prove the effect of centralM2 macrophages on prognosis, excluding the possibility of random fluctuations as shown in Table S1. OS was calculated from the time of surgery till death or the last follow-up visit (June 30, 2018), and DFS was calculated from the time of surgery till the relapse of disease. Univariate and multivariate analyses were performed by Cox proportional hazards regression models to estimate HR and $95 \%$ CI. Statistical significance was considered at $P<0.05$, and all statistical analyses were conducted using the SPSS statistical software package (version 16.0) and GraphPad Prism v7.0 for Windows.

\section{Results}

\section{Patient characteristics and multiplex quantitative immunofluorescence staining of NSCLC patient specimens}

To visualize the macrophage landscape in the microenvironment of NSCLC tumors, 137 patients with stage I-III NSCLC were enrolled. The clinicopathological features of the patients are summarized in Table 1. The median age at diagnosis was 59 years (range, 34-75 years). Among the patients, 77 were male (56.2\%) and 60 were female (43.8\%), and $93(67.9 \%)$ patients had adenocarcinoma and $44(32.1 \%)$ had squamous cell carcinoma. Fifty-nine (43.1\%) patients had EGFR mutations and 78 (56.9\%) had wild-type EGFR, which is similar to the known rate of genetic mutations in NSCLC patients in Asia. ${ }^{42}$ Sixty-five (47.4\%) patients had a history of smoking, and 81 (59.1\%) patients had lymphatic invasion. According to the criteria
Table I Characteristics of the patients at baseline

\begin{tabular}{|l|l|}
\hline Characteristic & All patients(N= I37) \\
\hline $\begin{array}{l}\text { Age } \\
\text { Median (range) }\end{array}$ & $59(34-75)$ \\
\hline $\begin{array}{l}\text { Gender } \\
\text { Female }\end{array}$ & $60(43.8 \%)$ \\
Male & $77(56.2 \%)$ \\
\hline EGFR status & \\
EGFR mutant & $59(43.1 \%)$ \\
EGFR wild type & $78(56.9 \%)$ \\
\hline Smoking history & $72(52.6 \%)$ \\
Never & $65(47.4 \%)$ \\
Former or current & \\
\hline Stage & $46(33.6 \%)$ \\
I & $46(33.6 \%)$ \\
II & $45(32.8 \%)$ \\
III & \\
\hline T & $112(81.8 \%)$ \\
I-2 & $25(18.2 \%)$ \\
\hline $3-4$ & $56(40.9 \%)$ \\
\hline N & $93(59.1 \%)$ \\
\hline 0 & $44(32.1 \%)$ \\
\hline Histologic diagnosis & \\
Adenocarcinoma & \\
\hline Squamous-cell carcinoma & \\
\hline
\end{tabular}

in the American Joint Committee on Cancer staging manual, 46 (33.6\%) patients had stage I cancer, 46 (33.6\%) had stage II, and 45 (32.8\%) had stage III. Multiplex quantitative immunofluorescence staining of pan-CK, CD68, CD163, PD-L1, and DAPI was performed on the NSCLC tissues. DAPI labels nuclei, CK was used to label tumor cells, CD68 labels macrophages, and CD163 labels M2 macrophages (Figure 1A). Then, the total number of macrophages, macrophage subtypes, density, and the distribution of macrophages within the tumor was analyzed.

\section{The correlation between macrophage distribution and tumor stages}

First, the distribution of macrophages in the NSCLC microenvironment was analyzed. The results showed that the number of macrophages in the tumor tissues accounted for $15.84 \%$ of all cells in tumor tissue ( $27.8 \%$ of all stroma cells). Among the macrophages, $36.68 \%$ were M1 and $63.32 \%$ were M2 (Figure 1B, Table 2); furthermore, $77.18 \%$ of macrophages 
A
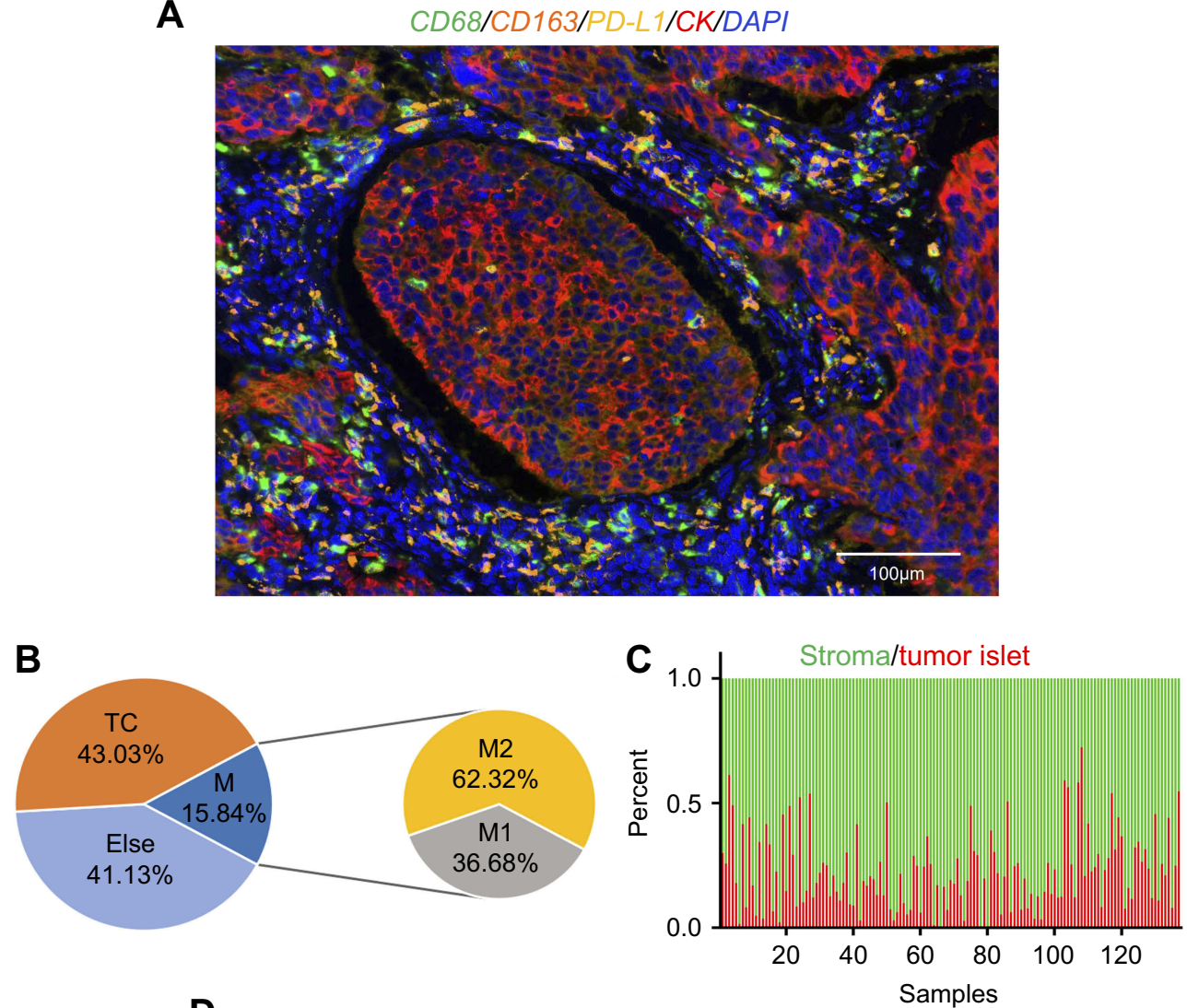

D

CD68/CD163/CK/DAPI

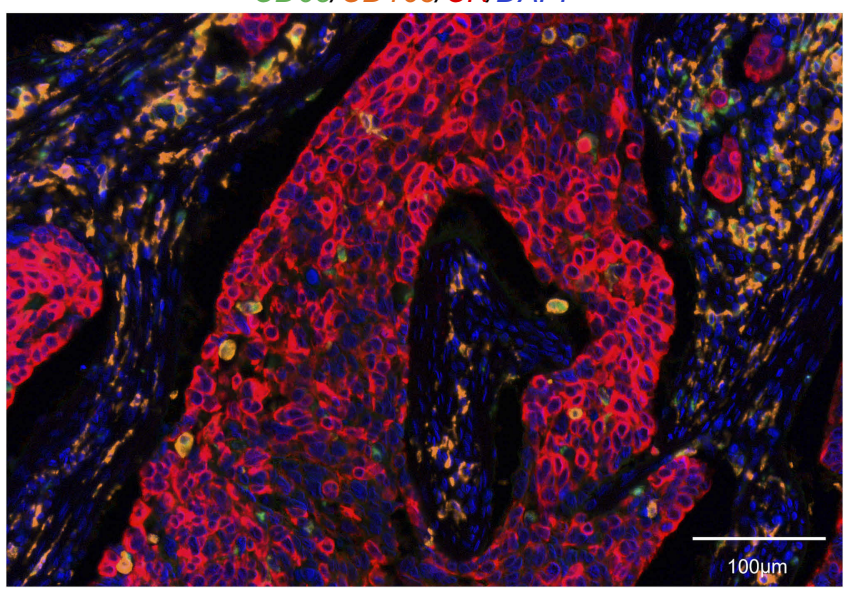

Figure I The macrophage landscape in the microenvironment of NSCLC. (A) A representative image using multiple quantitative fluorescence staining. Blue, DAPI; red, CK; green, CD68; orange, CDI63; yellow, PD-LI. Scale bars $=100 \mu \mathrm{m}$. (B) Quantity of cell subpopulations normalized to the total cell numbers in TIME. (C) Proportion of macrophage distribution in stroma (green) and tumor islets (red) of 137 patients in NSCLC microenvironment. Each column represents a patient. (D) A representative image of macrophages distributed in the tumor microenvironment. Blue, DAPI; red, CK; green, CD68; orange, CDI63. Scale bars $=100 \mu \mathrm{m}$.

Abbreviations: TC, tumor cells; M, total macrophages; MI, MI macrophages; M2, M2 macrophages; NSCLC, non-small-cell lung cancer; TIME, tumor immune microenvironment.

were located around the tumor interstitial region (these macrophages are defined as marginM), whereas $22.82 \%$ of the macrophages infiltrated the tumor islets (defined as centralM) (Figure 1C, 1D, Table 2). These results indicated that macrophages, especially the M2 type, are abundant in the microenvironment of lung cancer and are mostly distributed in the interstitial region of the tumor margin.
Then, we analyzed the relationship between macrophage number, distribution, and the stage of NSCLC. Although there was no significant change in overall macrophage infiltration (Figure 2A, 2D, 2G), as shown in Figure 2B-C, the amount of total M2 macrophages and the M2 polarization status $(\mathrm{M} 2 / \mathrm{M})$ significantly increased from stage I to stage III ( $P=0.005, P=0.02$, respectively). We also observed the sites 
Table 2 Cell proportion of different subtypes

\begin{tabular}{|l|l|}
\hline Cell type & Percent (\%) \\
\hline M/ALL & 15.84 \\
MI/ALL & 5.81 \\
M2/ALL & 10.03 \\
MI/M & 36.68 \\
M2/M & 63.32 \\
CentralM/M & 24.56 \\
MarginM/M & 75.44 \\
CenterMI/MI & 37.80 \\
MarginMI/MI & 62.20 \\
CenterM2/M2 & 16.89 \\
MarginM2/M2 & 83.11 \\
\hline
\end{tabular}

Abbreviations: $M$, total macrophages; $M 1$, total $M I$ macrophages; $M 2$, total $M 2$ macrophages; centralM, $M$ macrophages infiltrating into islets; marginM, $M$ macrophages in stroma; centralMI, MI macrophages infiltrating into islets; $\operatorname{marginMI}, \mathrm{MI}$ macrophages in stroma; centralM2, M2 macrophages infiltrating into islets; marginM2, M2 macrophages in stroma; ALL, total number of cells in tissue.

of macrophage infiltration in tumors of various stages. CentralM2 and marginM2 increased from state I to stage III (Figure 2E, 2H, $P=0.001, P=0.055$, respectively), and the M2 polarization status (M2/M) significantly increased from stage I to stage III in both tumor islet and tumor margin (Figure 2F, 2I, $P=0.004, P=0.05$, respectively). CentralM1 (M1 macrophages infiltrating into tumor islets) rapidly increased while marginM1 (M1 macrophages infiltrating in stroma) decreased in stage II, and then central and marginM1 both decreased in stage III (Figure 2E, 2H). The amounts of total macrophages, especially M2 macrophages, marginM2, and centralM2, closely correlated to the NSCLC stage, suggesting that M2 macrophages may play an important role in the development of NSCLC.

\section{The correlation between macrophages and pathological types, EGFR status, and smoking status}

Next, to investigate whether different clinicopathological features of NSCLC affect macrophage status, the relationships between macrophages and pathological types, EGFR status, and smoking status were analyzed.
A

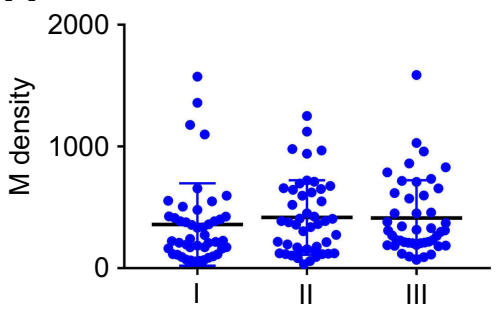

D
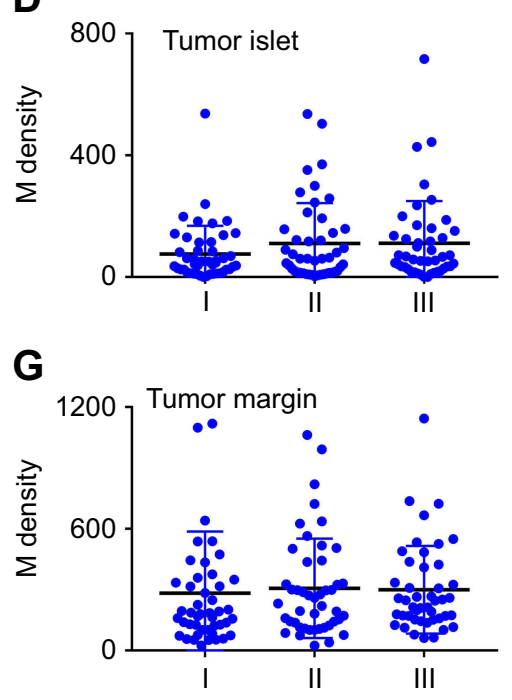

B

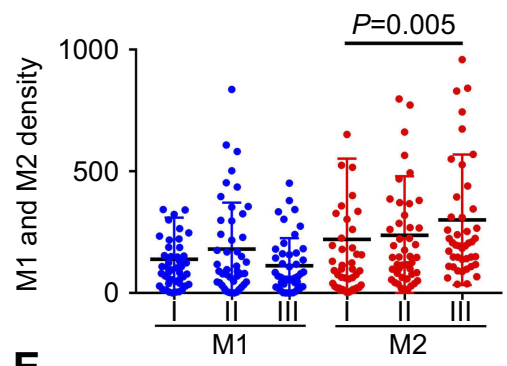

E

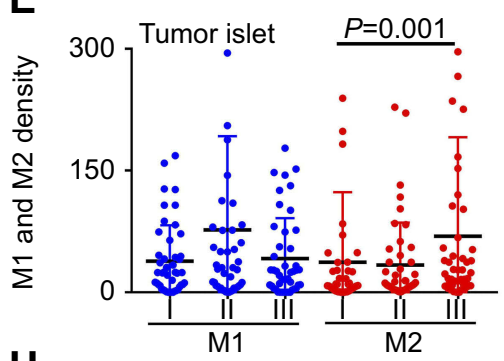

H

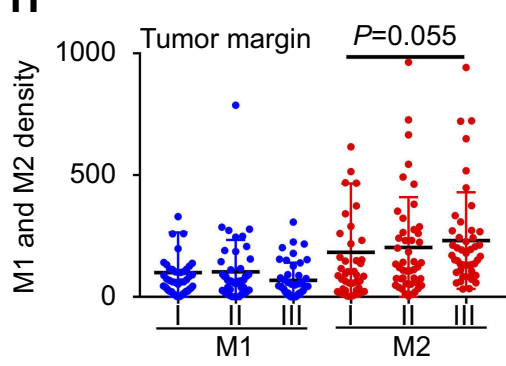

C

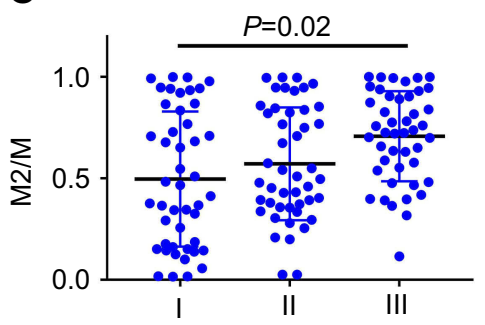

F Tumor islet

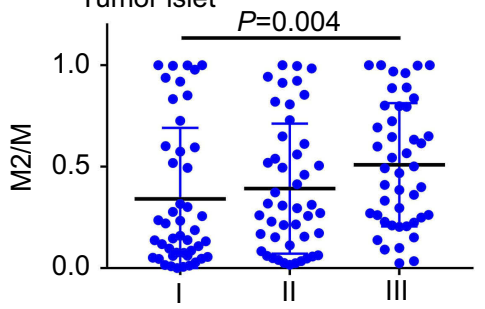

I Tumor margin

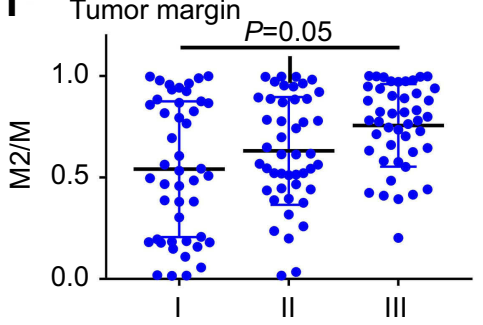

Figure 2 Distribution of macrophages in different tumor stages. (A-C) Represents the global distribution of macrophages. (D-F) Represents the distribution of macrophages in tumor islet. (G-I) Represents the distribution of macrophages in the stroma. Density $=$ cell number $/ 3.6 \times 10^{5}, \mathrm{~m}^{2} 2 . P<0.05$ indicates statistical significance. Abbreviations: $M$, total macrophages; MI, MI macrophages; M2, M2 macrophages. 
Compared to LUAD patients, patients with EGFR mutations, and non-smokers, higher densities of total macrophages were found in LUSC patients, patients with wild-type EGFR, and smokers, respectively $(P<0.05$, Figure 3A, 3D, 3G). Similar results were seen when $\mathrm{M} 1$ and $\mathrm{M} 2$ macrophages were analyzed (Figure 3B-C, 3E-F, 3H-I). Additionally, M2 polarization $(\mathrm{M} 2 / \mathrm{M})$ levels were nearly identical between the subgroups (Figure 3J, 3K, 3L). These results indicate that different features of macrophages in patients with different pathological characteristics may lead to diverse conditions in the immune microenvironment.

\section{Infiltration of macrophages and prognosis in NSCLC}

To examine the effect of macrophage infiltration on NSCLC prognosis, we analyzed the OS and DFS of the patients. The results showed that the OS of patients with high infiltration of M2 macrophages (M2 ${ }^{\text {more }}$ ) was significantly shorter than that of patients with low infiltration of M2 macrophages (M2 $\left.{ }^{\text {less }}\right)(P=0.003$, Figure $4 \mathrm{~B})$, whereas the total numbers of macrophages and M1 macrophages did not affect the OS (Figure 4A, 4C). Compared to patients with high infiltration of centralM and centralM2, patients with low infiltration of centralM $(P=0.003$,
A

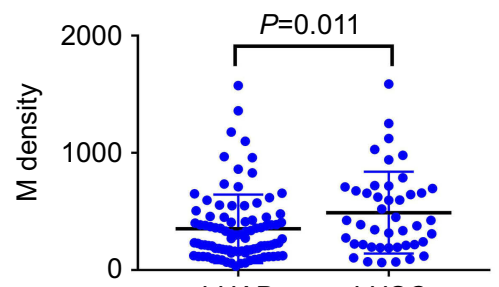

D

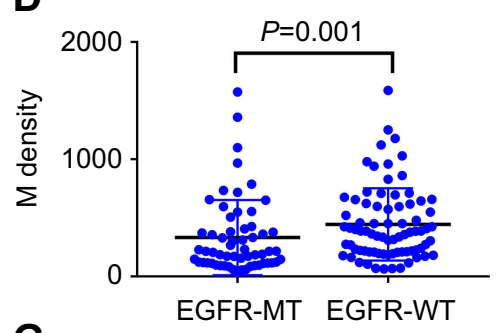

G

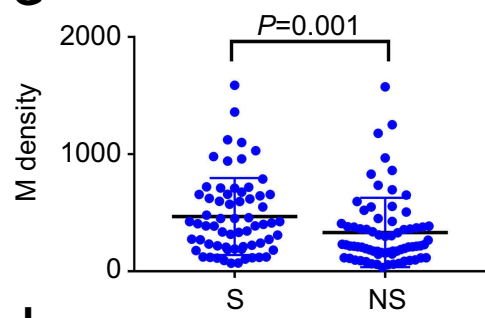

J

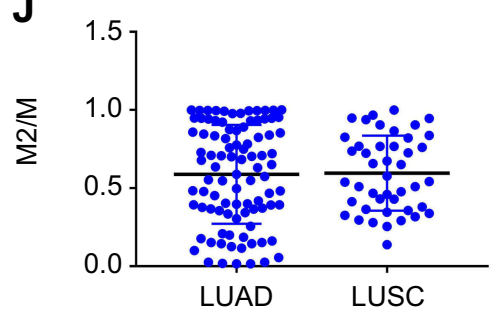

B

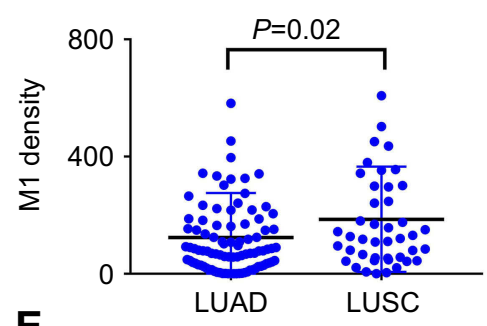

E

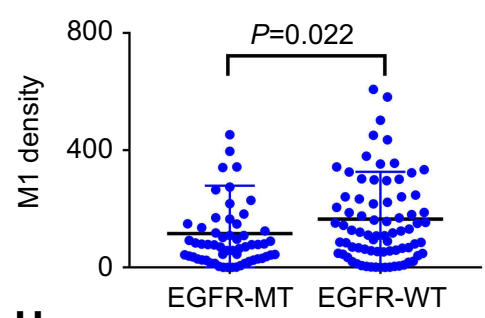

H
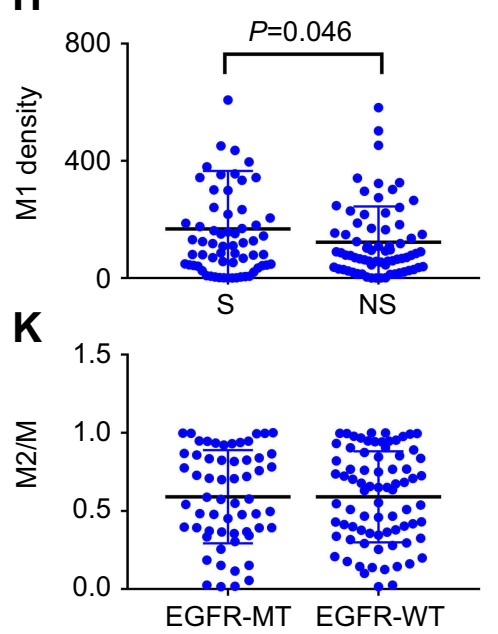

C

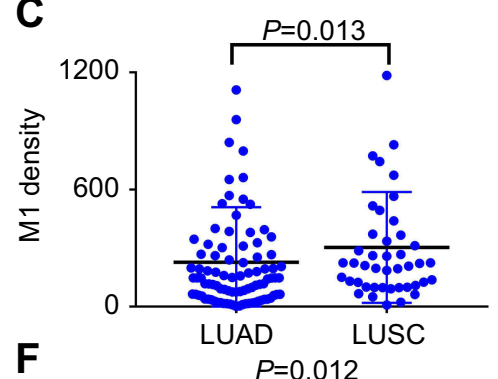

F
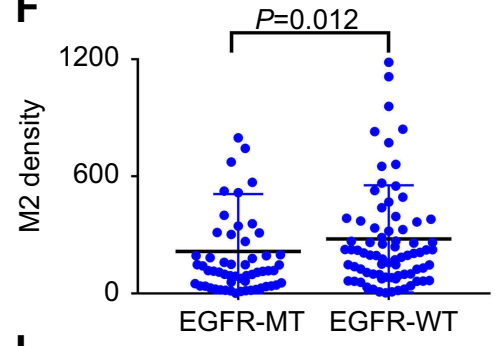

I

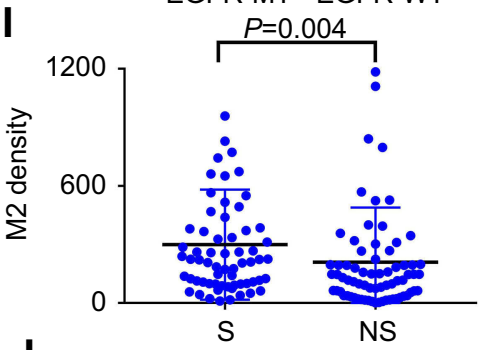

$\mathbf{L}$

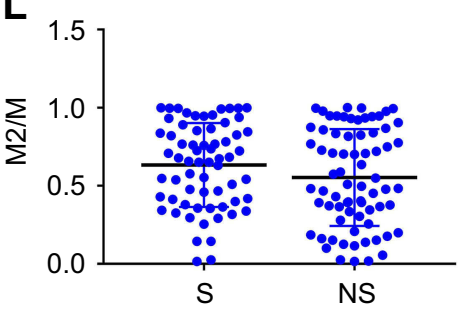

Figure 3 Quantity of macrophages in patients with different clinicopathological parameters. (A-C) Density of total macrophages (A), MI macrophages (B), M2 macrophages (C) in patients with different pathological types. (D-F) Density of total macrophages (D), MI macrophages (E), M2 macrophages (F) in patients with different EGFR status. (G-I) Density of total macrophages (G), MI macrophages (H), M2 macrophages (I) in patients with different smoking history. (J-L) Proportion of M2 macrophages to total macrophages in patients with different pathological types $(\mathbf{J})$, EGFR status $(\mathbf{K})$ and smoking history $(\mathbf{L})$. Density $=$ cell number/3.6 $\times 105, \mathrm{~m}^{2}$. $P<0.05$ indicates statistical significance.

Abbreviations: $M$, total macrophages; MI, MI macrophages; M2, M2 macrophages; M2/M, Proportion of M2 macrophages to total macrophages; LUAD, lung adenocarcinoma; LUSC, lung squamous cell carcinoma; EGFR-MT, EGFR mutation; EGFR-WT, EGFR wild-type; S, smoker; NS, nonsmokers. 
A
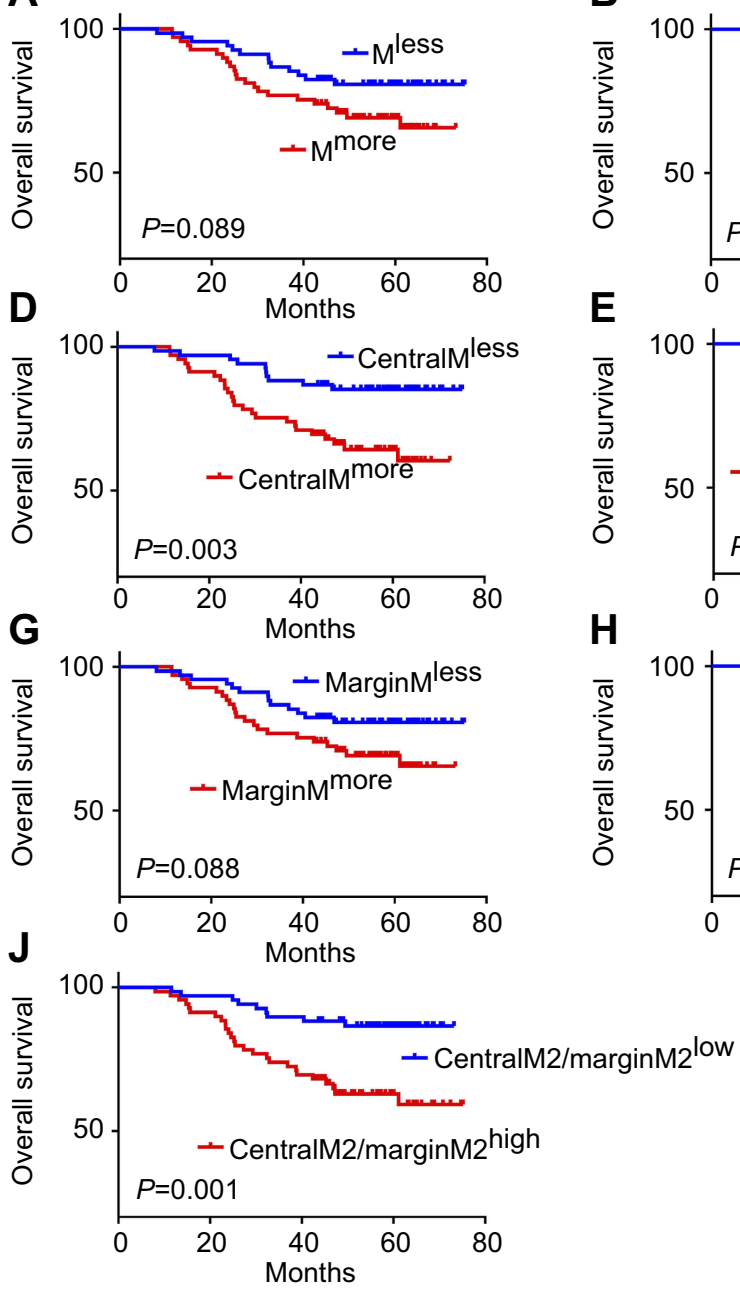

B
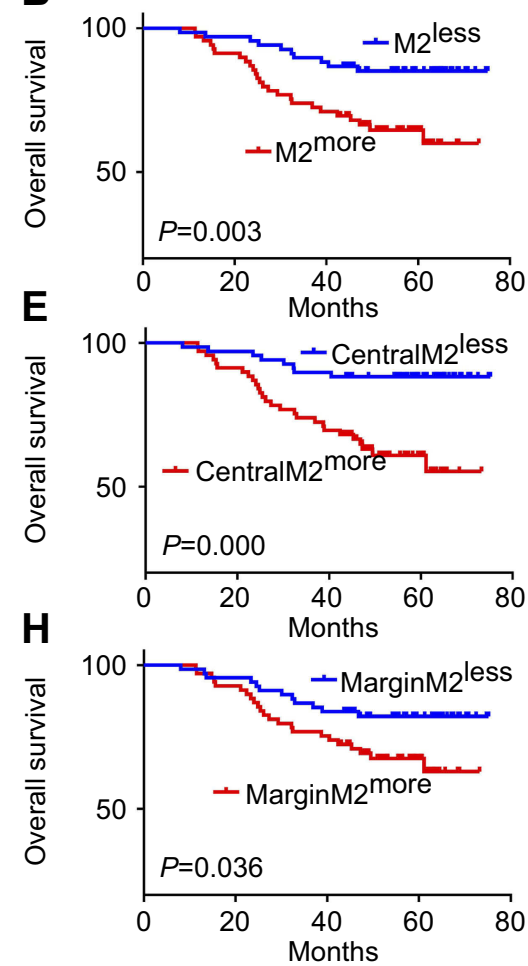
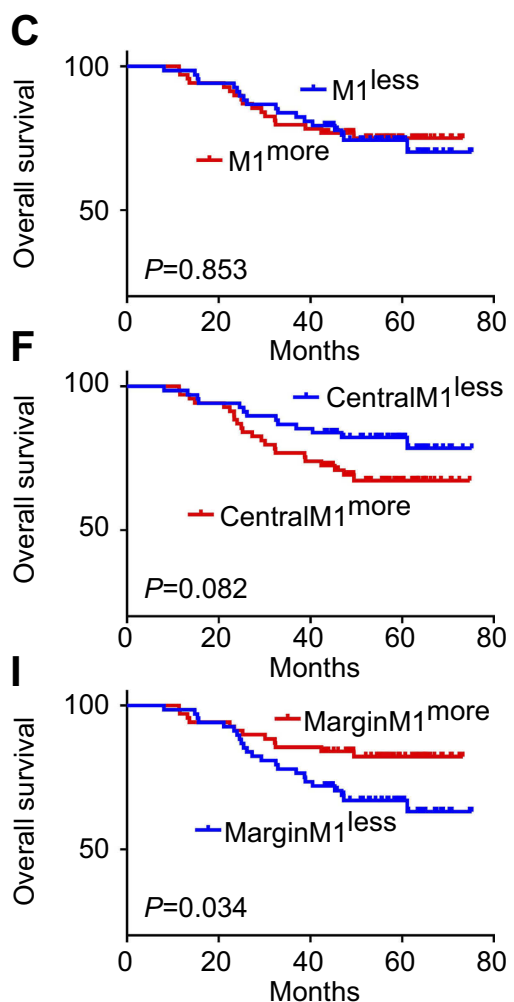

Figure 4 Overall survival (OS) analysis for macrophages of different polarization states and locations.

Notes: (A-C) Kaplan-Meier curve of the relationship between the total macrophages (M) (A), total M2 macrophages (M2) (B), total MI macrophages (MI) (C) and the OS of NSCLC patients. (D-F) Kaplan-Meier curve of the relationship between the macrophages infiltrating in tumor islets (centralM) (D), M2 macrophages infiltrating in tumor islets (centralM2) (E), MI macrophages infiltrating in tumor islets (centralMI) (F) and the OS of NSCLC patients. (G-I)Kaplan-Meier curve of the relationship between the macrophages in stroma (marginM) (G), M2 macrophages in stroma (marginM2) (H), MI macrophages in stroma (marginMI) (I) and the OS of NSCLC patients. (J)KaplanMeier curve of the relationship between the ratio of $\mathrm{M} 2$ macrophages infiltrating in tumor islets to $\mathrm{M} 2$ macrophages infiltrating in stroma (centralM2/marginM2) and the OS of NSCLC patients.less: low infiltration; more: high infiltration.

Figure 4D), especially centralM2 ( $P=0.000$, Figure 4E), had a better OS, but centralM1 did not affect the OS significantly $(P=0.082$, Figure $4 \mathrm{~F})$. MarginM1 did not affect the OS significantly ( $P=0.088$, Figure $4 \mathrm{G})$, while patients with high infiltration of marginM1 had a better OS than those with low infiltration of marginM1 $(P=0.034$, Figure $4 \mathrm{I})$. In addition, high infiltration of marginM2 also decreased the OS $(P=0.036$, Figure $4 \mathrm{H})$. To further clarify whether centralM2 or marginM2 has a more significant effect on the prognosis of NSCLC, we analyzed the correlation between the ratio of centralM2/marginM2 and the OS. Patients with centralM $2 /$ marginM $2^{\text {high }}$ had a worse OS than those with centralM2/marginM $2^{\text {low }}(P=0.001$,
Figure 4J), suggesting that centralM2 have a greater impact on the OS of NSCLC patients than marginM2.

Similarly, the analysis of DFS showed that patients with low infiltration of centralM ( $P=0.023$, Figure 5D), especially centralM2 ( $P=0.006$, Figure $5 \mathrm{E}$ ), had better DFS than patients with high infiltration of centralM and centralM2, and the patients with centralM2/marginM $2^{\text {high }}$ had worse DFS than those with centralM2/marginM $2^{\text {low }}(P=0.005$, Figure $5 \mathrm{~J})$. However, the total numbers of macrophages, M1 macrophages, M2 macrophages, the marginM, marginM1, marginM2, and centralM1 did not affect the DFS (Figure 5A-C, F-I). These results indicate that centralM2 has an important impact on the DFS of NSCLC patients. 


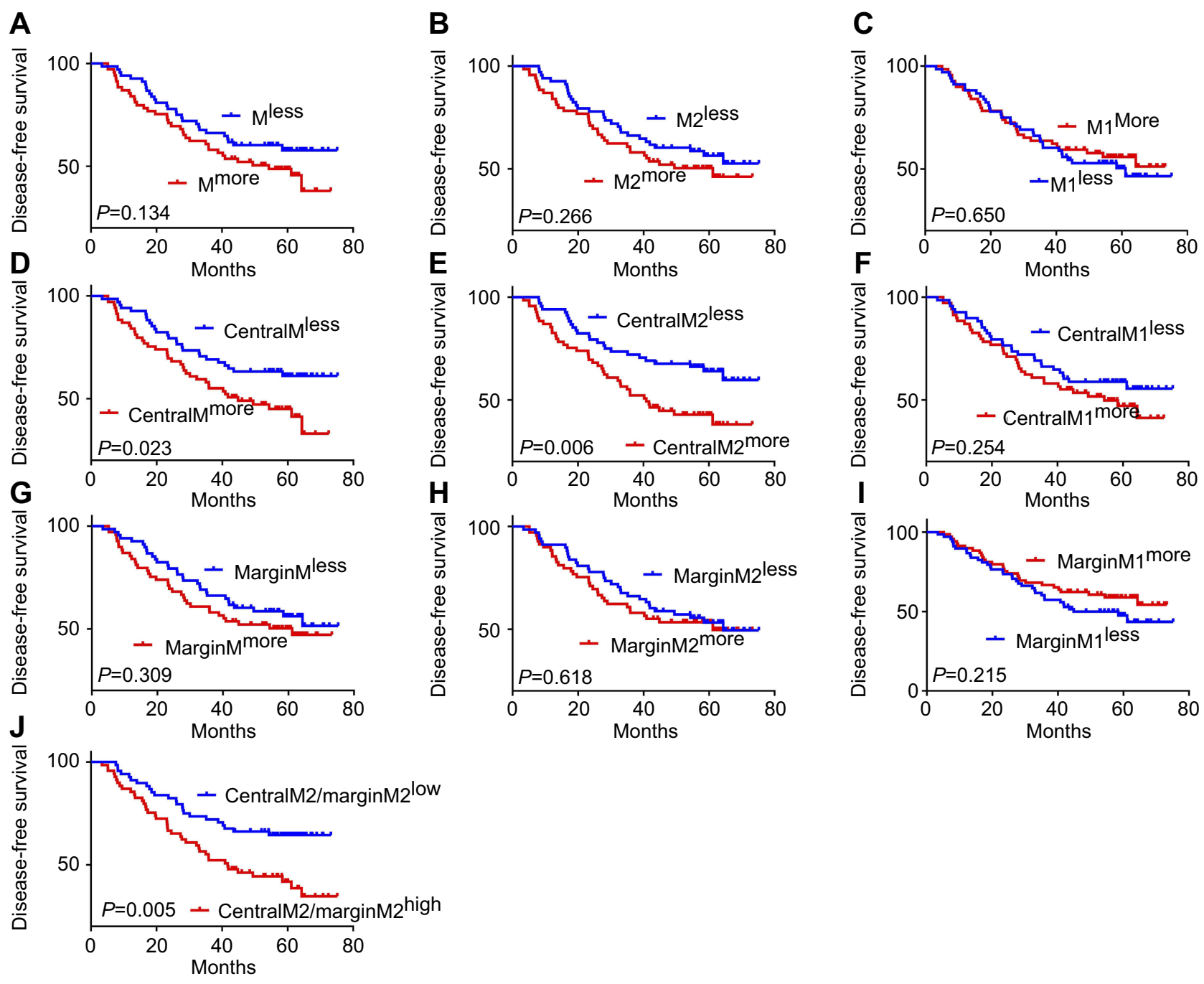

Figure 5 Disease-free survival analysis (DFS) for macrophages of different polarization states and locations.

Notes: (A-C) Kaplan-Meier curve of the relationship between the total macrophages (M) (A), total M2 macrophages (M2) (B), total MImacrophages (MI) (C) and the DFS. (D-F) Kaplan-Meier curve of the relationship between the macrophages infiltrating in tumor islets (centralM) (D), M2 macrophages infiltrating in tumor islets (centralM2) (E), MI macrophages infiltrating in tumor islets (centralMI) (F) and the DFS. (G-I) Kaplan-Meier curve of the relationship between the macrophages infiltrating in stroma $($ marginM) $(\mathbf{G})$, M2 macrophages infiltrating in stroma (marginM2) (H), MI macrophages infiltrating in stroma (marginMI) (I) and the DFS.(J)Kaplan-Meier curve of the relationship between the ratio of M2 macrophages infiltrating in tumor islets to M2 macrophages infiltrating in stroma (centralM2/marginM2) and the DFS. less: low infiltration; more: high infiltration.

Next, to determine whether the density of M1 and M2 macrophages is an independent factor of prognosis, univariable and multivariable analyses were carried out. Clinical characteristics and macrophage subtypes were subjected to univariable analysis, and the variables associated with OS or DFS at a significance level of $P<0.05$ were then subjected to the multivariate analysis (Table 3 , Table 4). The results revealed that the amount of centralM2 was an independent predictor of both OS and DFS (OS, HR $=2.859,95 \%$ CI $(1.125-7.262), P=0.027$; DFS, HR $=1.809,95 \%$ CI $(1.085-3.015), \quad P=0.023$; Table 3, Table 4). These results suggest that infiltration of M2 macrophages into the tumor islets has an important influence on the prognosis of NSCLC patients.

\section{Combined prognostic analysis of $M 2$ macrophages and tumor PD-LI}

To investigate whether M2 macrophages and tumor PD-L1 expression have a combined effect on NSCLC prognosis, the patients were divided into 4 groups according to the density of $\mathrm{M} 2$ macrophages and tumor PD-L1 expression: M2 ${ }^{\text {less }} \mathrm{PD}$ $\mathrm{L}^{-}{ }^{-}, \mathrm{M} 2{ }^{\text {less }} \mathrm{PD}-\mathrm{L} 1^{+}, \mathrm{M}_{2}{ }^{\text {more }} \mathrm{PD}-\mathrm{L} 1^{-}$, and $\mathrm{M} 2{ }^{\text {more }} \mathrm{PD}-\mathrm{L1}^{+}$. Patients with $\mathrm{M} 2^{\text {less }} \mathrm{PD}-\mathrm{L} 1^{-}$had the best $\mathrm{OS}$, while patients with $\mathrm{M} 2{ }^{\text {more }} \mathrm{PD}-\mathrm{L} 1^{+}$had the worst OS $(P=0.027$, Figure $6 \mathrm{~A})$. 
Table 3 Univariate and multivariate analysis for overall survival in 137 NSCLC patients

\begin{tabular}{|c|c|c|c|c|}
\hline & \multicolumn{2}{|l|}{ Univariate analysis } & \multicolumn{2}{|l|}{ Multivariate analysis } \\
\hline & HR $(95 \% \mathrm{Cl})$ & $P$-value & HR $(95 \% \mathrm{CI})$ & $P$-value \\
\hline Gender & $1.254(0.637-2.467)$ & 0.512 & & \\
\hline Age & I.I52 (0.593-0.593) & 0.677 & & \\
\hline Pathology & $0.858(0.4 \mid 2-1.787)$ & 0.682 & & \\
\hline EGFR & $0.837(0.426-1.647)$ & 0.607 & & \\
\hline Smoke history & $1.309(0.672-2.55)$ & 0.428 & & \\
\hline $\mathrm{T}$ & 2.654 (1.319-5.338) & 0.006 & $1.673(0.793-3.529)$ & 0.176 \\
\hline $\mathbf{N}$ & $5.102(1.976-13.177)$ & 0.001 & $4.053(1.555-10.563)$ & 0.004 \\
\hline CentralMI & $1.820(0.917-3.615)$ & 0.087 & & \\
\hline MarginMI & $0.494(0.245-0.993)$ & 0.048 & $0.7 I I(0.34-I .484)$ & 0.363 \\
\hline CentralM2 & 4.194 (1.897-9.27) & 0.000 & $2.859(1.125-7.262)$ & 0.027 \\
\hline MarginM2 & $2.152(1.069-4.329)$ & 0.032 & $1.029(0.465-2.276)$ & 0.944 \\
\hline
\end{tabular}

Notes: Bold values indicate statistical significance, $P<0.05$.

Abbreviations: centralMI, MI macrophages infiltrating into islets; marginMI, MI macrophages in stroma; centralM2, M2 macrophages infiltrating into islets; marginM2, $\mathrm{M} 2$ macrophages in stroma.

Table 4 Univariate and multivariate analysis for disease-free survival in I37 NSCLC patients

\begin{tabular}{|c|c|c|c|c|}
\hline & \multicolumn{2}{|l|}{ Univariate analysis } & \multicolumn{2}{|c|}{ Multivariate analysis } \\
\hline & HR $(95 \% \mathrm{CI})$ & $P$-value & HR $(95 \% \mathrm{CI})$ & $P$-value \\
\hline Gender & $0.877(0.539-1.428)$ & 0.598 & & \\
\hline Age & $1.001(0.614-1.631)$ & 0.998 & & \\
\hline Pathology & $0.783(0.454-1.349)$ & 0.378 & & \\
\hline EGFR & $1.258(0.773-2.048)$ & 0.356 & & \\
\hline Smoke history & $0.852(0.52|-| .39 \mid)$ & 0.521 & & \\
\hline $\mathrm{T}$ & $1.499(0.842-2.67)$ & 0.169 & & \\
\hline $\mathbf{N}$ & 2.591 (1.499-4.479) & 0.001 & $2.325(1.335-4.05)$ & 0.003 \\
\hline centralMI & $1.275(0.783-2.076)$ & 0.33 & & \\
\hline marginMI & $0.762(0.467-1.244)$ & 0.278 & & \\
\hline centralM2 & $2.092(1.264-3.463)$ & 0.004 & $1.809(1.085-3.015)$ & 0.023 \\
\hline marginM2 & $1.183(0.726-1.927)$ & 0.499 & & \\
\hline
\end{tabular}

Notes: Bold values indicate statistical significance, $P<0.05$.

Abbreviations: centralMI, MI macrophages infiltrating into islets; marginMI, MI macrophages in stroma; centralM2, M2 macrophages infiltrating into islets; marginM2, $M 2$ macrophages in stroma.

However, no statistically significant differences in DFS were seen among these 4 groups (Figure 6B).

Next, analysis of correlation between the prognosis and the combination of centralM2 and tumor PD-L1 was performed. The OS and DFS in the centralM2 ${ }^{\text {less }} \mathrm{PD}-\mathrm{L1}^{-}$group were the best, while the centralM2 ${ }^{\text {more }} \mathrm{PD}-\mathrm{L1}^{+}$group had the worst $\mathrm{OS}$ and DFS ( $P=0.002,0.034$, respectively, Figure 6C, 6D). Moreover, the prognosis of the centralM $2^{\text {less }} \mathrm{PD}-\mathrm{L}^{+}$group was better than that of the centralM2 ${ }^{\text {more }} \mathrm{PD}-\mathrm{L}^{-}{ }^{-}$group, suggesting that the centralM2 macrophages may be more important than tumor PD-L1 expression in NSCLC prognosis. Taken together, these results suggest that the combined analysis of macrophages and tumor PD-L1 could improve the accuracy of prognosis prediction.

\section{Discussion}

In this study, we found that M2 macrophages are important immune cells in TIME of NSCLC, that high infiltration of centralM2 was an independent predictor of poor OS and DFS, and that the analysis of macrophage subtype and tumor PD-L1 in combination could improve the accuracy of prognosis prediction in NSCLC. The multiplex quantitative fluorescence staining method used in this study could not only distinguish the tumor and stroma areas 
A

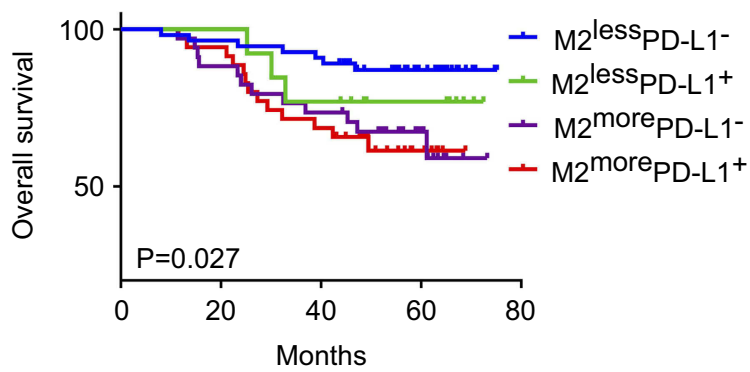

C

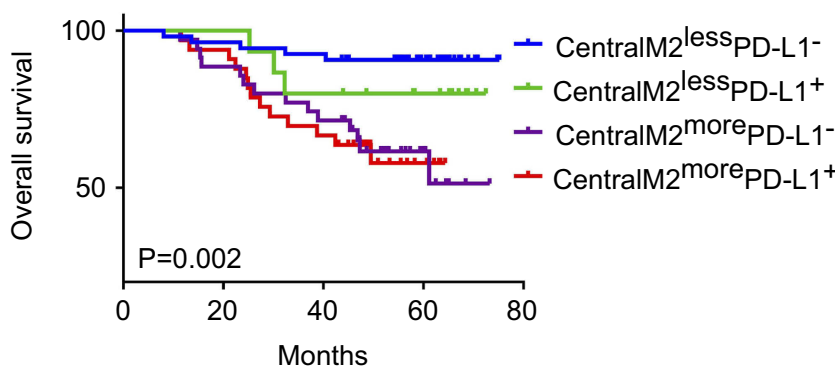

B

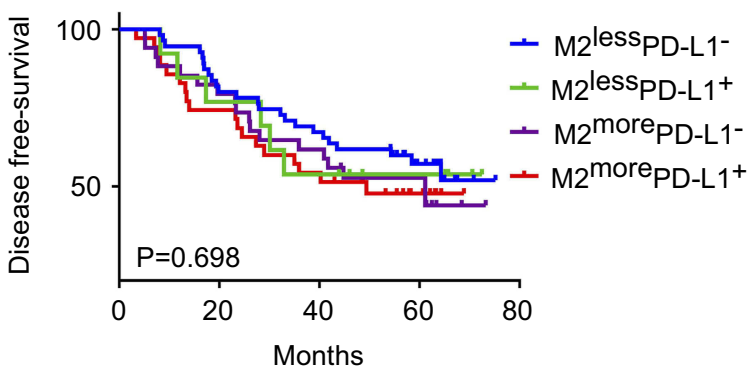

D

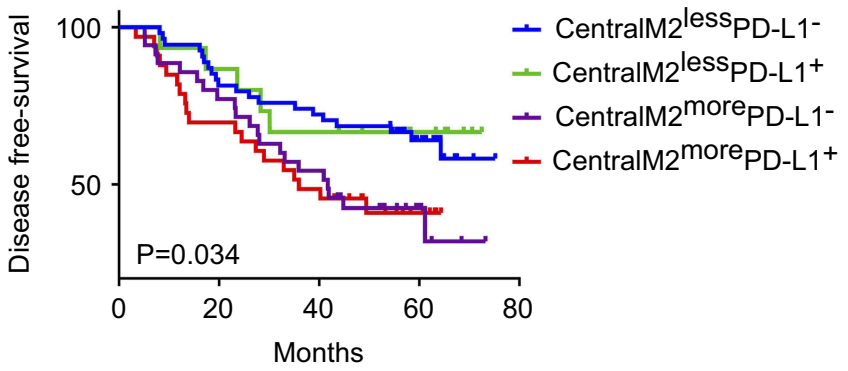

Figure 6 Prognostic analysis of M2 macrophages and tumor PD-LI combination of NSCLC. (A) Kaplan-Meier curve of the relationship between the combination of M2 macrophages and tumor PD-LI, and the overall survival (OS). (B) Kaplan-Meier curve of the relationship between the combination of M2 macrophages and tumor PD-LI and the disease-free survival (DFS). (C) Kaplan-Meier curve of the relationship between the combination of centralM2 macrophages and tumor PD-LI, and the OS. (D) Kaplan-Meier curve of the relationship between the combination of marginM2 macrophages and tumor PD-LI, and the DFS.

Notes: $\mathrm{M}^{\text {less }}{ }^{\mathrm{PD}}-\mathrm{LI}^{-}$: low infiltration of $\mathrm{M} 2$ macrophages and tumor PD-LI negative; $\mathrm{M} 2^{\text {less }} \mathrm{PD}-\mathrm{LI}^{+}$: low infiltration of $\mathrm{M} 2$ macrophages and tumor PD-LI positive;

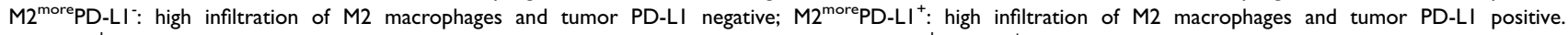
centralM2 ${ }^{\text {less }} \mathrm{PD}-\mathrm{LI}^{-}$: low infiltration ofcentralM2 macrophages and tumor PD-LI negative; centralM2 $2^{\text {less }} \mathrm{PD}-\mathrm{LI} \mathrm{I}^{+}$: low infiltration of centralM2 macrophages and tumor PD$\mathrm{LI}$ positive; centralM2 ${ }^{\text {more }} \mathrm{PD}-\mathrm{LI}^{-}$: high infiltration of centralM2 macrophages and tumor PD-LI negative; centralM2 ${ }^{\text {more }}{ }^{\mathrm{PD}}-\mathrm{LI}^{+}$: high infiltration of centralM2 macrophages and tumor PD-LI positive.

clearly, but also allowed us to identify and count macrophages accurately by simultaneously labeling multiple markers on the same tissue slice. Thus, this method is extremely advantageous for TIME research and allows for more detailed studies than classical immunohistochemistry methods.

Our results provide important new information about macrophages in the TIME. First, macrophages, especially the M2 type, were abundant in the microenvironment of NSCLC and were mostly distributed in the interstitial region of the tumor margin. This is consistent with previous studies. Second, the polarization of macrophages from M1 to M2 during the development of NSCLC is a dynamic process. We found that macrophage number, polarization, and infiltration sites were different in various tumor stages, which may illustrate the development of macrophages from tumor suppression to tumor promotion in cancer development. In the early stages of tumor development, M1 macrophages increasingly infiltrate to the tumor islets to suppress tumor progression. However, as the tumor develops, the M1 macrophages gradually transform into M2 macrophages. In the late stages, most of the macrophages are polarized to M2 and promote tumor progression. Third, high infiltration of M2 macrophages, especially centralM2, was associated with poor prognosis in NSCLC. As mentioned above, the results from previous studies examining the prognostic role of macrophages were controversial. Several recent studies have shown that the polarization status and localization of macrophages, but not the total amount of macrophage infiltration, were related to the prognosis of lung cancer. ${ }^{22,23}$ Jurgita et al reported that tumor islet-infiltrating M1 macrophages and the number of total M2 macrophages were independent predictors of NSCLC patient survival, ${ }^{30}$ while $\mathrm{Li}$ et al found that a high number of M2 macrophages in tumor stroma, but not in tumor islets or alveolar space, was a significant prognostic factor for DFS in NSCLC patients. ${ }^{28}$ We considered that limitations of traditional immunohistochemistry might be one of the reasons for the inconsistent conclusions, which is dependent on the manual judgment for the identification of cell types, dividing tissue regions and counting cells. Therefore, to perform a more precise and objective analysis, we used multiplex quantitative immunofluorescence staining, of 
which the counting process is quantitative and automatic, to examine the macrophage landscape in the TIME. We demonstrated that high M2 infiltration led to poor prognosis, and that the effect of M2 macrophages infiltrating into the tumor islets was stronger than that of M2 macrophages in the tumor margin. This suggests that the closer the M2 cells are to the tumor cells, the stronger the effect they have on tumor development. In general, our results suggest that centralM2 plays an important role in the prognosis of NSCLC, a result that is different from those of previous studies.

M2 macrophages are known to promote tumor development through direct and indirect mechanisms. M2 can directly promote angiogenesis, tumor matrix remodeling, and invasion by secreting multiple proangiogenic mediators, ${ }^{7-12,18}$ growth factors, and proteolytic enzymes. ${ }^{13,14}$ M2 macrophages can also inhibit T-cell proliferation and activation by secreting immunosuppressive factors such as CCL22, IL-10, and TGF- $\beta$, and by recruiting regulatory $\mathrm{T}$ cells (Treg) to tumor tissues. Therefore, M2 can indirectly promote tumor development by dampening the antitumor immune response. ${ }^{15}$ In this study, centralM2 had a stronger effect on tumor development than marginM2. However, the reasons for this are unclear. One of the possible mechanisms is that the cytokines and inhibitory inflammatory factors secreted by the centralM2 may be closer to the tumor cells, which allows for higher concentrations of these factors to interact with the tumor cells for longer durations. Moreover, direct interaction between molecules on the surface of the centralM2 and tumor cells may also contribute to malignant biological behavior in tumor cells. Further studies are needed to define these mechanisms.

We also found that patients with different infiltration levels of M2 macrophages and PD-L1 expression had different prognoses. The OS and DFS in centralM2 ${ }^{\text {less }} \mathrm{PD}-\mathrm{L}^{-}{ }^{-}$group were the best, while the centralM2 ${ }^{\text {more }} \mathrm{PD}-\mathrm{L}^{+}$group had the worst OS and DFS. Therefore, the analysis of M2 macrophage infiltration and PD-L1 expression in combination could further increase the accuracy of prognostic prediction for NSCLC. Macrophages and tumor PD-L1 may affect each other, reflecting the complexity of the TIME.

While analyzing the association of macrophages and various clinical subgroups, it was found that the infiltration of macrophages in patients with LUSC, wild-type EGFR, and a history of smoking was higher than in patients with LUAD, EGFR mutations, and non-smokers. These results indicate strong correlations between the amount of macrophages and clinical subgroups, EGFR status, and smoking status of NSCLC patients. Several studies have shown that
NSCLC patients who are smokers and have wild-type EGFR were able to benefit the most from anti-PD-1 therapy. ${ }^{43-47}$ Therefore, anti-PD-1 therapy may be the most beneficial for patients with abundant macrophage infiltration. Our findings provide a new potential strategy for priority population selection for anti-PD-1/PD-L1 therapy.

\section{Conclusion}

Tumor islet-infiltrating M2 macrophages influence the prognosis of NSCLC patients, and the analysis of M2 macrophages and tumor PD-L1 in combination may enhance the accuracy of prognostic predictions. This study provides a new understanding of macrophages in the development of NSCLC and provides a means of prognostic prediction through the analysis of the macrophage landscape in NSCLC.

\section{Ethical approval}

All procedures performed in studies involving human participants were in accordance with the ethical standards of the institutional research committee and with the 1964 Helsinki Declaration and its later amendments or comparable ethical standards. The study was approved by the Ethics Committee of China Medical University (No. 2017-236), and all patients have signed the informed consent.

\section{Abbreviation list}

NSCLC, non-small-cell lung cancer; LUSC, lung squamous cell carcinoma; LUAD, lung adenocarcinoma; OS, overall survival; DFS, disease-free survival; TIME, tumor immune microenvironment; PD-1, programmed death receptor 1; PD-L1, programmed cell death-Ligand 1; EGFR, epithelial growth factor receptor; AJCC, the American Joint Committee on Cancer.

\section{Acknowledgments}

This work was supported by National Science and Technology Major Project of the Ministry of Science and Technology of China (No. 2017ZX09304025), the National Natural Science Foundation of China (No. 81602098, 31770963), Science and Technology Plan Project of Liaoning Province (No.2016007010, 2015020457), the Key Research and Development Program of Shenyang (No. 17-230-9-01) and foundation for Selected Overseas Chinese Scholar 2015. 


\section{Author contributions}

All authors contributed to data analysis, drafting or revising the article, gave final approval of the version to be published, and agree to be accountable for all aspects of the work.

\section{Disclosure}

The authors report no conflicts of interest in this work.

\section{References}

1. Bray F, Ferlay J, Soerjomataram I, Siegel RL, Torre LA, Jemal A. Global cancer statistics 2018: GLOBOCAN estimates of incidence and mortality worldwide for 36 cancers in 185 countries. CA Cancer J Clin. 2018;68(6):394-424. doi:10.3322/caac.21492

2. Torre LA, Bray F, Siegel RL, Ferlay J, Lortet-Tieulent J, Jemal A. Global cancer statistics, 2012. CA Cancer J Clin. 2015;65(2):87-108. doi: $10.3322 /$ caac. 21262

3. Langer CJ, Gadgeel SM, Borghaei H, et al. Carboplatin and pemetrexed with or without pembrolizumab for advanced, non-squamous non-small-cell lung cancer: a randomised, phase 2 cohort of the openlabel KEYNOTE-021 study. Lancet Oncol. 2016;17(11):1497-1508. doi:10.1016/S1470-2045(16)30498-3

4. Borghaei H, Langer CJ, Gadgeel S, et al. 24-month overall survival from KEYNOTE-021 Cohort G: pemetrexed and carboplatin with or without pembrolizumab as first-line therapy for advanced nonsquamous non-small cell lung cancer. $J$ Thorac Oncol. 2019;14(1):124129.

5. Brahmer J, Reckamp KL, Baas P, et al. Nivolumab versus docetaxel in advanced squamous-cell non-small-cell lung cancer. $N$ Engl J Med. 2015;373(2):123-135.

6. Morrison C. Immuno-oncologists eye up macrophage targets. Nat Rev Drug Discov. 2016;15(6):373-374.

7. Murdoch C, Muthana M, Coffelt SB, Lewis CE. The role of myeloid cells in the promotion of tumour angiogenesis. Nat Rev Cancer. 2008;8(8):618-631.

8. Nakao S, Kuwano T, Tsutsumi-Miyahara C, et al. Infiltration of COX-2-expressing macrophages is a prerequisite for IL-1 betainduced neovascularization and tumor growth. $J$ Clin Invest. 2005;115(11):2979-2991.

9. Lin EY, Li JF, Bricard G, et al. Vascular endothelial growth factor restores delayed tumor progression in tumors depleted of macrophages. Mol Oncol. 2007;1(3):288-302.

10. Chen P, Huang Y, Bong R, et al. Tumor-associated macrophages promote angiogenesis and melanoma growth via adrenomedullin in a paracrine and autocrine manner. Clin Cancer Res. 2011;17 (23):7230-7239. doi:10.1158/1078-0432.CCR-11-1354

11. Guruvayoorappan C. Tumor versus tumor-associated macrophages: how hot is the link? Integr Cancer Ther. 2008;7(2):90-95. doi:10.1177/1534735408319060

12. Du R, Lu KV, Petritsch C, et al. HIF1alpha induces the recruitment of bone marrow-derived vascular modulatory cells to regulate tumor angiogenesis and invasion. Cancer Cell. 2008;13(3):206-220. doi:10.1016/j.ccr.2008.01.034

13. Gocheva V, Wang HW, Gadea BB, et al. IL-4 induces cathepsin protease activity in tumor-associated macrophages to promote cancer growth and invasion. Genes Dev. 2010;24(3):241-255. doi:10.1101/gad.1874010

14. Gil-Bernabe AM, Ferjancic S, Tlalka M, et al. Recruitment of monocytes/macrophages by tissue factor-mediated coagulation is essential for metastatic cell survival and premetastatic niche establishment in mice. Blood. 2012;119(13):3164-3175. doi:10.1182/blood-2011-08-376426

15. Kryczek I, Zou L, Rodriguez P, et al. B7-H4 expression identifies a novel suppressive macrophage population in human ovarian carcinoma. $J$ Exp Med. 2006;203(4):871-881. doi:10.1084/jem.20050930
16. Takanami I, Takeuchi K, Kodaira S. Tumor-associated macrophage infiltration in pulmonary adenocarcinoma: association with angiogenesis and poor prognosis. Oncology. 1999;57(2):138-142. doi:10.1159/000012021

17. Chen JJ, Yao PL, Yuan A, et al. Up-regulation of tumor interleukin-8 expression by infiltrating macrophages: its correlation with tumor angiogenesis and patient survival in non-small cell lung cancer. Clin Cancer Res. 2003;9(2):729-737.

18. Leek RD, Lewis CE, Whitehouse R, Greenall M, Clarke J, Harris AL. Association of macrophage infiltration with angiogenesis and prognosis in invasive breast carcinoma. Cancer Res. 1996;56 (20):4625-4629.

19. Lissbrant IF, Stattin P, Wikstrom P, Damber JE, Egevad L, Bergh A. Tumor associated macrophages in human prostate cancer: relation to clinicopathological variables and survival. Int J Oncol. 2000;17 (3):445-451.

20. Shimura S, Yang G, Ebara S, Wheeler TM, Frolov A, Thompson TC. Reduced infiltration of tumor-associated macrophages in human prostate cancer: association with cancer progression. Cancer Res. 2000;60 (20):5857-5861.

21. Khorana AA, Ryan CK, Cox C, Eberly S, Sahasrabudhe DM. Vascular endothelial growth factor, CD68, and epidermal growth factor receptor expression and survival in patients with Stage II and Stage III colon carcinoma: a role for the host response in prognosis. Cancer. 2003;97(4):960-968. doi:10.1002/cncr.11152

22. Johnson SK, Kerr KM, Chapman AD, et al. Immune cell infiltrates and prognosis in primary carcinoma of the lung. Lung Cancer. 2000;27 (1):27-35.

23. Toomey D, Smyth G, Condron C, et al. Infiltrating immune cells, but not tumour cells, express FasL in non-small cell lung cancer: no association with prognosis identified in 3-year follow-up. Int $J$ Cancer. 2003;103(3):408-412. doi:10.1002/ijc.10836

24. Yuan A, Hsiao YJ, Chen HY, et al. Opposite effects of M1 and M2 macrophage subtypes on lung cancer progression. Sci Rep. 2015;5:14273. doi:10.1038/srep 14273

25. Sun Y, Xu S. Tumor-associated CD204-positive macrophage is a prognostic marker in clinical stage i lung adenocarcinoma. Biomed Res Int. 2018;2018:8459193.

26. Zhang B, Yao G, Zhang Y, et al. M2-polarized tumor-associated macrophages are associated with poor prognoses resulting from accelerated lymphangiogenesis in lung adenocarcinoma. Clinics (Sao Paulo). 2011;66(11):1879-1886.

27. Hirayama S, Ishii G, Nagai K, et al. Prognostic impact of CD204positive macrophages in lung squamous cell carcinoma: possible contribution of Cd204-positive macrophages to the tumor-promoting microenvironment. $J$ Thorac Oncol. 2012;7(12):1790-1797. doi:10.1097/JTO.0b013e3182745968

28. Li Z, Maeda D, Yoshida M, et al. The intratumoral distribution influences the prognostic impact of CD68- and CD204-positive macrophages in non-small cell lung cancer. Lung Cancer. 2018;123:127-135. doi:10.1016/j.lungcan.2018.07.015

29. Kim DW, Min HS, Lee KH, et al. High tumour islet macrophage infiltration correlates with improved patient survival but not with EGFR mutations, gene copy number or protein expression in resected non-small cell lung cancer. Br J Cancer. 2008;98(6):1118-1124. doi:10.1038/sj.bjc. 6604256

30. Jackute J, Zemaitis M, Pranys D, et al. Distribution of M1 and M2 macrophages in tumor islets and stroma in relation to prognosis of non-small cell lung cancer. BMC Immunol. 2018;19(1):3. doi:10.1186/s12865-018-0241-4

31. Gordon SR, Maute RL, Dulken BW, et al. PD-1 expression by tumourassociated macrophages inhibits phagocytosis and tumour immunity. Nature. 2017;545(7655):495-499. doi:10.1038/nature22396

32. Dhupkar P, Gordon N, Stewart J, Kleinerman ES. Anti-PD-1 therapy redirects macrophages from an M2 to an M1 phenotype inducing regression of OS lung metastases. Cancer Med. 2018;7(6):26542664. doi: $10.1002 / \mathrm{cam} 4.1518$ 
33. Guo W, Zhang F, Shao F, et al. PD-L1 expression on tumor cells associated with favorable prognosis in surgically resected esophageal squamous cell carcinoma. Hum Pathol. 2019;84: 291-298.

34. Troiano G, Caponio VCA, Zhurakivska K, et al. High PD-L1 expression in the tumour cells did not correlate with poor prognosis of patients suffering for oral squamous cells carcinoma: a meta-analysis of the literature. Cell Prolif. 2019;52(2):e12537.

35. Yu H, Chen Z, Ballman KV, et al. Correlation of PD-L1 expression with tumor mutation burden and gene signatures for prognosis in early-stage squamous cell lung carcinoma. J Thorac Oncol. 2019;14 (1):25-36.

36. Okuma Y, Hishima T, Kashima J, Homma S. Correction to: high PDL1 expression indicates poor prognosis of HIV-infected patients with non-small cell lung cancer. Cancer Immunol Immunother. 2018;67 (9):1477-1479. doi:10.1007/s00262-018-2215-z

37. Xia H, Shen J, Hu F, et al. PD-L1 over-expression is associated with a poor prognosis in Asian non-small cell lung cancer patients. Clin Chim Acta. 2017;469:191-194. doi:10.1016/j.cca.2017.02.005

38. Berntsson J, Eberhard J, Nodin B, Leandersson K, Larsson AH, Jirstrom K. Expression of programmed cell death protein 1 (PD-1) and its ligand PD-L1 in colorectal cancer: relationship with sidedness and prognosis. Oncoimmunology. 2018;7(8):e1465165. doi:10.1080/ 2162402X.2018.1490854

39. Wang Y, Zhu C, Song W, Li J, Zhao G, Cao H. PD-L1 expression and $\mathrm{CD} 8(+) \mathrm{T}$ cell infiltration predict a favorable prognosis in advanced gastric cancer. J Immunol Res. 2018;2018:4180517. doi:10.1155/ $2018 / 4180517$
40. Zhu Q, Cai MY, Chen CL, et al. Tumor cells PD-L1 expression as a favorable prognosis factor in nasopharyngeal carcinoma patients with pre-existing intratumor-infiltrating lymphocytes. Oncoimmunology. 2017;6(5):e1312240. doi:10.1080/2162402X.2017.1312240

41. Jung HI, Jeong D, Ji S, et al. Overexpression of PD-L1 and PD-L2 is associated with poor prognosis in patients with hepatocellular carcinoma. Cancer Res Treat. 2017;49(1):246-254. doi:10.4143/crt.2016.066

42. Shi Y, Au JS, Thongprasert S, et al. A prospective, molecular epidemiology study of EGFR mutations in Asian patients with advanced non-smallcell lung cancer of adenocarcinoma histology (PIONEER). J Thorac Oncol. 2014;9(2):154-162. doi:10.1097/JTO.0000000000000033

43. Garassino MC, Gelibter AJ, Grossi F, et al. Italian nivolumab expanded access program in nonsquamous non-small cell lung cancer patients: results in never-smokers and EGFR-mutant patients. $J$ Thorac Oncol. 2018;13(8):1146-1155.

44. Borghaei H, Paz-Ares L, Horn L, et al. Nivolumab versus docetaxel in advanced nonsquamous non-small-cell lung cancer. $N$ Engl J Med. 2015;373(17):1627-1639.

45. Kobayashi H, Omori S, Nakashima K, et al. Response to the treatment immediately before nivolumab monotherapy may predict clinical response to nivolumab in patients with non-small cell lung cancer. Int J Clin Oncol. 2017;22(4):690-697.

46. Gettinger S, Rizvi NA, Chow LQ, et al. Nivolumab monotherapy for first-line treatment of advanced non-small-cell lung cancer. $J$ Clin Oncol. 2016;34(25):2980-2987.

47. Lee CK, Man J, Lord S, et al. Checkpoint inhibitors in metastatic EGFR-mutated non-small cell lung cancer-a meta-analysis. J Thorac Oncol. 2017;12(2):403-407. 


\section{Supplementary material}

Table SI COX analysis of continuous variables

\begin{tabular}{|l|l|l|l|l|}
\hline \multirow{2}{*}{} & \multicolumn{2}{|l|}{ OS } & \multicolumn{2}{l|}{ DFS } \\
\cline { 2 - 5 } & HR (95\% CI) & P-value & HR (95\% CI) & P-value \\
\hline M2 & $1.088(0.993-1.192)$ & $0.07 I$ & $1.016(0.937-1.103)$ & 0.696 \\
CentralM2 & $1.571(1.237-1.996)$ & $\mathbf{0 . 0 0 0}$ & $1.438(1.155-1.790)$ & $\mathbf{0 . 0 0 1}$ \\
MarginM2 & $1.061(0.943-1.194)$ & 0.327 & $0.973(0.873-1.085)$ & 0.628 \\
cM2/mM2 & $1.92(1.276-2.889)$ & $\mathbf{0 . 0 0 2}$ & $1.971(1.402-2.771)$ & $\mathbf{0 . 0 0 0}$ \\
\hline
\end{tabular}

Notes: Bold values indicate statistical significance, $P<0.05$.

Abbreviations: centralM2, M2 macrophages infiltrating into islets; marginM2, M2 macrophages in stroma; $\mathrm{cM} 2, \mathrm{M} 2 \mathrm{macrophages}$ infiltrating into islets; mM2, M2 macrophages in stroma.

\section{Publish your work in this journal}

Cancer Management and Research is an international, peer-reviewed open access journal focusing on cancer research and the optimal use of preventative and integrated treatment interventions to achieve improved outcomes, enhanced survival and quality of life for the cancer patient.
The manuscript management system is completely online and includes a very quick and fair peer-review system, which is all easy to use. Visit http://www.dovepress.com/testimonials.php to read real quotes from published authors. 